\title{
EFFECTIVE TREE SCATTERING AT L-BAND
}

\author{
Mehmet Kurum ${ }^{(1)}$, Peggy E. O'Neill ${ }^{(1)}$, Roger H. Lang ${ }^{(2)}$, Alicia T. Joseph ${ }^{(1)}$, Michael H. Cosh \\ ${ }^{(3)}$, and Thomas J. Jackson ${ }^{(3)}$, \\ ${ }^{(1)}$ Hydrological Sciences Branch / Code 614.3 NASA Goddard Space Flight Center, \\ Greenbelt, MD 20771 USA, Mehmet.Kurum@nasa.gov \\ (2) The George Washington University, Dept. of Elect. \& Computer Engineering, \\ Washington, DC, 20052 USA \\ (3) Hydrology \& Remote Sensing Laboratory, USDA ARS, Beltsville, MD 20705 USA
}

\section{INTRODUCTION}

For routine microwave Soil Moisture (SM) retrieval through vegetation, the tau-omega [1] model [zero-order Radiative Transfer (RT) solution] is attractive due to its simplicity and eases of inversion and implementation. It is the model used in baseline retrieval algorithms for several planned microwave space missions, such as ESA's Soil Moisture Ocean Salinity (SMOS) mission (launched November 2009) and NASA's Soil Moisture Active Passive (SMAP) mission (to be launched 2014/2015) [2 and 3]. These approaches are adapted for vegetated landscapes with effective vegetation parameters tau and omega by fitting experimental data or simulation outputs of a multiple scattering model [4-7]. The model has been validated over grasslands, agricultural crops, and generally light to moderate vegetation.

As the density of vegetation increases, sensitivity to the underlying SM begins to degrade significantly and errors in the retrieved SM increase accordingly. The zero-order model also loses its validity when dense vegetation (i.e. forest, mature corn, etc.) includes scatterers, such as branches and trunks (or stalks in the case of corn), which are large with respect to the wavelength. The tau-omega model (when applied over moderately to densely vegetated landscapes) will need modification (in terms of form or effective parameterization) to enable accurate characterization of vegetation parameters with respect to specific tree types, anisotropic 
canopy structure, presence of leaves and/or understory. More scattering terms (at least up to first-order at L-band) should be included in the RT solutions for forest canopies [8]. Although not really suitable to forests, a zero-order tau-omega model might be applied to such vegetation canopies with large scatterers, but that equivalent or effective parameters would have to be used [4]. This requires that the effective values (vegetation opacity and single scattering albedo) need to be evaluated (compared) with theoretical definitions of these parameters.

In a recent study [9], effective vegetation opacity of coniferous trees was compared with two independent estimates of the same parameter. First, a zero-order RT model was fitted to multiangular microwave emissivity data in a least-square sense to provide "effective" vegetation optical depth as done in spaceborne retrieval algorithms. Second, a ratio between radar backscatter measurements with a corner reflector under trees and in an open area was calculated to obtain "measured" tree propagation characteristics. Finally, the "theoretical" propagation constant was determined by forward scattering theorem using detailed measurements of size/angle distributions and dielectric constants of the tree constituents (trunk, branches, and needles). Results indicated that the "effective" attenuation values are smaller than but of similar magnitude to both the "theoretical" and "measured" values.

This study will complement the previous work [9] and will focus on characterization of effective scattering albedo by assuming that "effective" vegetation opacity is same as "theoretical" opacity. The resultant effective albedo will not be the albedo of single forest canopy element anymore, but it becomes a global parameter, which depends on all the processes taking place within the canopy including multiple scattering as described below.

\section{APPROACH}

The zero-order radiative transfer model [1] is given by

$$
e_{p}^{(0)}(\theta)=\left[1-\gamma_{p}^{2}(\theta) R_{s p}(\theta)\right]-\omega_{p}(\theta)\left[1+\gamma_{p}(\theta) R_{s p}(\theta)\right]\left[1-\gamma_{p}(\theta)\right]
$$


where the ambient temperatures of the vegetation layer and the ground are assumed to be same, $R_{s p}(\theta)$ is the microwave reflectivity of the forest floor, $\gamma_{p}(\theta)$ is the vegetation transmissivity which is parameterized as $\gamma_{p}(\theta)=\exp \left(-\tau_{p} \sec \theta\right)$ where $\tau_{p}$ is the vegetation opacity or optical thickness and $\theta$ is the incidence angle, and $\omega_{p}(\theta)$ is the single scattering albedo. The polarization $p$ can be horizontal (h) or vertical (v).

In case of a forest canopy, the scattering from large vegetation components such as branches and trunk is significant and the first-order radiative transfer model [8] describes emission and scattering processes better within the vegetation canopy and is given by:

$$
e_{p}^{(1)}(\theta)=e_{p}^{(0)}(\theta)+\Omega_{p}(\theta)
$$

where the parameter $\Omega_{p}(\theta)$ denotes the additional scattering contribution to the zero-order model. It represents the emission from the ground and the vegetation layer that is singlescattered from tree trunks, branches, and leaves (or needles).

In this paper, we will fit the zero-order model to the first-order model with an effective scattering albedo under assumption that assuming that "effective" vegetation opacity is same as "theoretical" opacity, i.e.,

$$
e_{p}^{(0)}\left(\bar{\omega}_{p}, \gamma_{p}, R_{s p}\right)=e_{p}^{(1)}\left(\omega_{p}, \gamma_{p}, R_{s p}\right)
$$

The effective scattering albedo is then obtained by:

$$
\bar{\omega}_{p}(\theta)=\omega_{p} k\left(\omega_{p}, \gamma_{p}, R_{s p}\right)
$$

where the proportionality factor is given by

$$
k\left(\omega_{p}, \gamma_{p}, R_{s p}\right)=1-\frac{\Omega_{p}(\theta) / \omega_{p}(\theta)}{\left[1+\gamma_{p}(\theta) R_{s p}(\theta)\right]\left[1-\gamma_{p}(\theta)\right]}
$$


As seen in (5), effective single scattering albedo depends on all the processes taking place within the canopy and both canopy and ground as a function of polarization and incidence angle. In this paper, the parameter $k\left(\omega_{p}, \gamma_{p}, R_{s p}\right)$ will evaluated against $1.4 \mathrm{GHz}$ brightness temperature measurements acquired over deciduous/coniferous trees by a truck-mounted microwave instrument system called ComRAD [10].

\section{REFERENCES}

[1] T. Mo, B. Choudhury, T. Schmugge, J. Wang, and T. A. Jackson, "Model for Microwave Emission from Vegetation Covered Fields," Journal of Geophysical Research, vol. 87, no. C13, pp. 11,229-11,238, Dec. 1982.

[2] Y. H. Kerr, P. Waldteufel, J. P. Wigneron, S. Delwart, F. Cabot, J. Boutin, M. J. Escorihuela, J. Font, N. Reul, C. Gruhier, S. E. Juglea, M. R. Drinkwater, A. Hahne, M. Martin-Neira, and S. Mecklenburg, "The SMOS Mission: New Tool for Monitoring Key Elements of the Global Water Cycle," Proceedings of the IEEE, vol. 98, no. 5, pp. 666 - 687, May 2010.

[3] D. Entekhabi, E. Njoku, P. E. O’Neill, K. Kellogg, W. Crow, W. Edelstein, J. Entin, S. Goodman, T. Jackson, J. Johnson, J. Kimball, J. Piepmeier, R. Koster, N. Martin, K. McDonald, M. Moghaddam, S. Moran, R. Reichle, J.C. Shi, M. Spencer, S. Thrman, L. Tsang, and V. Jakob, "The Soil Moisture Active and Passive (SMAP) Mission," Proceedings of the IEEE, vol. 98, no. 5, pp. 704 - 716, May 2010.

[4] Jackson T. J., "Measuring surface soil moisture using passive microwave remote sensing," Hydrological Processes, 7:139152. 1993.

[5] P. Ferrazolli, L. Guerriero, and J. P. Wigneron, "Simulating L-band Emission of Forests in View of Future Satellite Applications," IEEE Transactions on Geoscience and Remote Sensing, vol. 40, no. 12, pp. 2700-2708, Dec. 2002.

[6] E. Njoku, E. Jackson, V. Lakshmi, T. Chan, and S. Nghiem, "Soil Moisture Retrieval from AMSR-E," IEEE Transactions on Geoscience and Remote Sensing, vol. 41, no. 2, pp. 215-229, Feb. 2003.

[7] J. P. Wigneron, Y. H. Kerr, P. Waldteufel, K. Saleh, M. J. Escorihuela, P. Richaume, P. Ferrazzoli, P. de Rosnay, R. Gurney, J.-C. Calvet, J. P. Grant, M. Guglielmetti, B. Hornbuckle, C. Mätzler, T. Pellarin, and M. Schwank, "L-band Microwave Emission of the Biosphere (L-MEB) Model: Description and Calibration against Experimental Data Sets over Crop Fields," Remote Sensing of Environment, vol. 107, no. 4, pp. 639-655, Apr. 2007.

[8] M. Kurum, R. H. Lang, P. O’Neill, A Joseph, T. Jackson, and M. Cosh, “A First-Order Radiative Transfer Model for Microwave Radiometry of Forest Canopies at L-band”, in press, IEEE Transcation on Geoscience and Remote Sensing, 2010.

[9] M. Kurum, P. E. O’Neill, R. H. Lang, A. T. Joseph, M. H. Cosh, and T. J. Jackson, "Characterization of forest opacity using multi-angular emission and backscatter data", in Proceedings, International Geosciences and Remote Sensing Symposium, Honolulu, Hawaii (USA), July 25 - 30, 2010.

[10] P.E. O’Neill, R. H. Lang, M. Kurum, A. T. Joseph, T. J. Jackson, M. H. Cosh, and R. Nelson, “ComRAD active/passive microwave measurements of tree canopies," in Proc. Int. Geosci. Remote Sens. Symp., Barcelona, Spain, Jul. 2007, pp. 14201423. 